\title{
Schlafen11 Expression Is Associated With the Antitumor Activity of Trabectedin in Human Sarcoma Cell Lines
}

\author{
JUNYA IWASAKI $^{1,2}$, TOSHIHARU KOMORI ${ }^{1}$, FUMIO NAKAGAWA ${ }^{1}$, \\ HIDEKI NAGASE $^{1}$, JUNJI UCHIDA ${ }^{1}$, KENICHI MATSUO $^{3}$ and YOSHIHIRO UTO ${ }^{2}$ \\ ${ }^{1}$ Applied Pharmacology Section, Pharmacology Laboratory, Taiho Pharmaceutical Co., Ltd., Tokushima, Japan; \\ ${ }^{2}$ Graduate School of Technology, Industrial and Social Sciences, Tokushima University, Tokushima, Japan; \\ ${ }^{3}$ Pharmacology Laboratory, Taiho Pharmaceutical Co., Ltd., Tsukuba, Japan
}

\begin{abstract}
Background/Aim: Trabectedin is a DNAdamaging agent and has been approved for the treatment of patients with advanced soft tissue sarcoma. Schlafen 11 (SLFN11) was identified as a dominant determinant of the response to DNA-damaging agents. The aim of the study was to clarify the association between SLFN11 expression and the antitumor activity of trabectedin. Materials and Methods: The antitumor activity of trabectedin was evaluated under different expression levels of SLFN11 regulated by RNA interference and CRISPR-Cas 9 systems, and the combined antitumor activity of ataxia telangiectasia and Rad3-related protein kinase (ATR) inhibitor and trabectedin in sarcoma cell lines using in vitro a cell viability assay and in vivo xenograft models. Results: SLFN11-knockdown cell lines had a lower sensitivity to trabectedin, compared to parental cells. ATR inhibitor enhanced the antitumor activity of trabectedin in SLFN11-knockdown cells and in a SLFN11-knockout xenograft model. Conclusion: SLFN11 expression might be a key factor in the antitumor activity of trabectedin.
\end{abstract}

Trabectedin (Yondelis ${ }^{\circledR}$, ecteinascidin-743, ET-743) is a marine-derived natural product that has been approved for the treatment of patients with advanced soft tissue sarcoma and relapsed platinum-sensitive ovarian cancer in combination with liposomal doxorubicin $(1,2)$. Ongoing studies suggest that trabectedin is also effective against other solid malignancies, including breast cancer (3).

This article is freely accessible online.

Correspondence to: Yoshihiro Uto, Graduate School of Technology, Industrial and Social Sciences, Tokushima University, 2-1, Minamijosanjima-cho, Tokushima, 770-8506, Japan. Tel: +81 886567514, Fax: +81 886567514, e-mail. uto.yoshihiro@tokushima-u.ac.jp

Key Words: DNA repair, anticancer drug, sarcoma, Schlafen11, trabectedin.
Previous studies have shown a peculiar aspect of the mechanism of action of trabectedin. Trabectedin showed a decreased activity (from 2- to 10-fold) in nucleotide excision repair (NER)-deficient cells, compared to NER-proficient cells $(4,5)$. DNA-bound trabectedin is thought to prevent the correction of DNA lesions through transcription-coupled NER (TC-NER) by creating cytotoxic ternary complexes with DNA-binding proteins of the NER system, such as XPG. On the other hand, NER-deficient cells show an increased sensitivity to platinum drugs, such as cisplatin (46). Homologous recombination repair (HRR) has been shown to be important for trabectedin, since HRR-deficient cells were 50- to 100-times more sensitive to trabectedin (7, 8). The lack of HRR has been associated with the persistence of unrepaired DNA double-strand breaks (DSBs) during the S phase of the cell cycle and apoptosis (7). Moreover, the inhibition of the cell-cycle checkpoints that are activated in response to trabectedin might also prove useful to increase drug efficacy $(9,10)$. In response to replicative damage, ataxia telangiectasia and Rad3-related protein kinase (ATR) plays a major role in coordinating cell-cycle progression and DNA repair $(11,12)$. The loss of the S-phase checkpoint by ATR inhibitors causes the unscheduled firing of replication origins in S-phase and the induction of DSBs $(13,14)$. At present, a total of 39 different gene fusions have been identified in 15 different tumor types, accounting for approximately $20 \%$ of all soft tissue sarcomas (15). Furthermore, trabectedin interacts with fused genes and inhibits the expressions of oncogenes, which are upregulated by gene fusions such as EWS-FLI1 and FUSCHOP $(16,17)$.

Recently, Schlafen 11 (SLFN11) was discovered during bioinformatics analyses of cancer cell databases and was identified as a dominant determinant of cell response to some widely used anticancer drugs, including topoisomerase (Top) 1 inhibitors, Top2 inhibitors, alkylating agents, and DNA synthesis inhibitors $(18,19)$. The importance of SLFN11 in drug sensitivity has recently been extended to include 
Ewing's sarcoma (20). SLFN11 influences the response of patients with ovarian, non-small cell lung, or colorectal cancers $(18,19)$, and inhibits checkpoint maintenance and homologous recombination by removing replication protein A (RPA) from single-stranded DNA (21). Recent reports have shown that SLFN11 is recruited to stressed replication forks carrying extended RPA filaments where it blocks replication independently of HRR (22).

In this study, a correlation analysis comparing the antitumor activity of trabectedin and SLFN11 expression in sarcoma cell lines was performed and demonstrated the importance of SLFN11 expression as a key factor in the antitumor activity of trabectedin in sarcoma cell lines and xenograft models. Furthermore, the usefulness of combination therapies to overcome the decreased antitumor activity of trabectedin in cells with low SLFN11 expression levels was evaluated.

\section{Materials and Methods}

Cell lines. The human sarcoma cell lines Yamato-SS and Aska-SS were kindly provided by Dr. Norifumi Naka (Osaka International Cancer Institute, Osaka, Japan) and Dr. Kazuyuki Ito (Nozaki Tokushukai Hospital, Osaka, Japan); SYO-1 was kindly provided by Dr. Akira Kawai (National Cancer Center, Tokyo, Japan); HT-1080, KYM-1, NY, RD, SCCH-196, RKN, SKN and HuO9N2 were purchased from the Japanese Collection of Research Bioresources (Osaka, Japan); Hs 925.T, KHOS/NP, KHOS-240S, SK-LMS-1, KHOS-312H, SW 872, SW 982, MES-SA, U-2 OS, SK-ES-1, SJSA1, SJCRH30 and RD-ES were purchased from ATCC (Manassas, VA, USA); ESS-1 was purchased from DSMZ (Brunswick, Germany); and A673, HOS, MG-63, G-292 clone A141B1 and Saos-2 were purchased from KAC Co., Ltd. (Kyoto, Japan). All the cells were maintained according to the supplier's protocols.

Chemicals. Trabectedin was provided by PharmaMar (Madrid, Spain). VE-821 (http://www.selleckchem.com/products/ve821.html) was purchased from Selleck Chemicals (Houston, TX, USA). Berzosertib (VE-822; https://www.medchemexpress.com/VE822.html) was purchased from MedChemExpress (Monmouth Junction, NJ, USA). 5-Aza-2'-deoxycytidine (decitabine) was purchased from Sigma-Aldrich Co., LLC (St. Louis, MO, USA).

Antibodies. Antibodies against SLFN11 (sc-374339), and CHK1 (sc8408) were obtained from Santa Cruz Biotechnology (Dallas, TX, USA). Antibodies against phospho-Ser317-CHK1 (\#2344), phospho-Ser345-CHK1 (\#2348), phospho-Ser139-H2AX (\#9718), and GAPDH (\#2118) were obtained from Cell Signaling Technology (Danvers, MA, USA). Horseradish peroxidaseconjugated secondary antibodies to mouse or rabbit $\mathrm{IgG}$ were obtained from Cell Signaling Technology.

Cell viability assay. Cell viability was measured using the crystal violet staining method and CellTiter-Glo 2.0 Assay (Promega, Fitchburg, WI, USA). Approximately $2-5 \times 10^{3}$ cells were plated per well on a 96-well plate. The cells were treated with a range of drug concentrations for $72 \mathrm{~h}$. After adding the CellTiter-Glo reagent, luminescence readings were obtained using a VersaMax Absorbance
Microplate Reader (Molecular Devices, San Jose, CA, USA) and FlexStation3 (Molecular Devices). The 50\% and 75\% inhibitory concentrations $\left(\mathrm{IC}_{50}\right.$ and $\mathrm{IC}_{75}$ ) were calculated using an XLfit (Fit model 205, ITOCHU Techno-Solutions Co., Tokyo, Japan).

Quantitative RT-PCR. For the TaqMan array cards and the TaqMan gene expression assay (Thermo Fisher Scientific, Waltham, MA, USA) experiments, RNA was collected from 30 sarcoma cell lines using an RNeasy kit (Qiagen, Venlo, the Netherlands). Complementary DNA (cDNA) was synthesized using High-Capacity cDNA Reverse Transcription Kits (Thermo Fisher Scientific). The reaction was carried out following the methods for use of TaqMan Universal Master Mix II (2x) (Thermo Fisher Scientific), and ACTB and $G A P D H$ were used as the reference genes.

siRNA transfection. Cells $\left(1-3 \times 10^{5}\right)$ were plated in 6-well plates. The siRNA for human SLFN11 and negative control (Stealth RNAi siRNA, HSS132188 and 12935300) were products of Invitrogen (Carlsbad, CA, USA). siRNA (5 nmol/l) was transfected into each cell with Lipofectamine RNAiMAX Reagent (Invitrogen) according to the manufacturer's instructions.

Western blotting. Total protein was extracted from whole cells using a cellular protein extraction buffer containing protease and phosphatase inhibitors. The protein contents were measured using a Pierce ${ }^{\circledR}$ BCA Protein Assay Kit (Bio-Rad, Hercules, CA, USA). Protein extracts were separated using SDS-PAGE polyacrylamide gels and electroblotted onto a PVDF membrane (Bio-Rad). The blotted membrane was blocked with Blocking One and Blocking One P (Nacalai Tesque, Kyoto, Japan). The primary antibodies were diluted in Can Get Signal Immunoreaction Enhancer Solution 1 (TOYOBO, Osaka, Japan) at a 1:1,000 dilution for SLFN11, phospho-Ser317CHK1, phospho-Ser345-CHK1, CHK1, and phospho-Ser139-H2A.X and a 1:5000 dilution for GAPDH. The secondary antibody (Cell Signaling Technology) was diluted in Can Get Signal Immunoreaction Enhancer Solution 2 at a 1:2,000 dilution. Signals were detected by using a ChemiDoc Touch (Bio-Rad).

Establishment of SLFN11-knockout cells. To delete the SLFN11 gene, we used Edit-R CRISPR RNA (crRNA), which was designed to target the human SLFN11 gene (CM-01674-01-0002 and CM01674-02-0002) (Dharmacon, Lafayette, CO, USA). crRNA, Edit$\mathrm{R}$ trans-activating CRISPR RNA, and SMARTCas9 (Puro ${ }^{\circledR}$ ) Expression Plasmid were co-transfected into SW 872 cells according to the instruction manual. Approximately 1 week after transfection, the cells were cultured in the presence of puromycin (1.5-2.5 $\mu \mathrm{g} / \mathrm{ml})$. Approximately 3-4 weeks later, puromycin-resistant clones were then selected and allowed to expand. A lack of SLFN11 protein expression in the SW 872 knockout cells was confirmed using western blotting.

In vivo experiments. Five-week-old male $\mathrm{BALB} / \mathrm{c}$ nude mice were purchased from CLEA Japan, Inc. (Tokyo, Japan) and were housed under specific pathogen-free conditions. Following a quarantine period of about 1 week, tumor fragments (approximately $2 \mathrm{~mm}$ diameter) were implanted subcutaneously via an implant needle in the vicinity of the right hindmost rib of the nude mice. The length $(\mathrm{mm})$ and width $(\mathrm{mm})$ were measured, and the tumor volume $\left(\mathrm{TV}, \mathrm{mm}^{3}\right)$ for each fragment was calculated using the following formula: $\mathrm{TV}=($ length $) \times(\text { width })^{2} / 2$. When the TV reached $100-200 \mathrm{~mm}^{3}$, the 


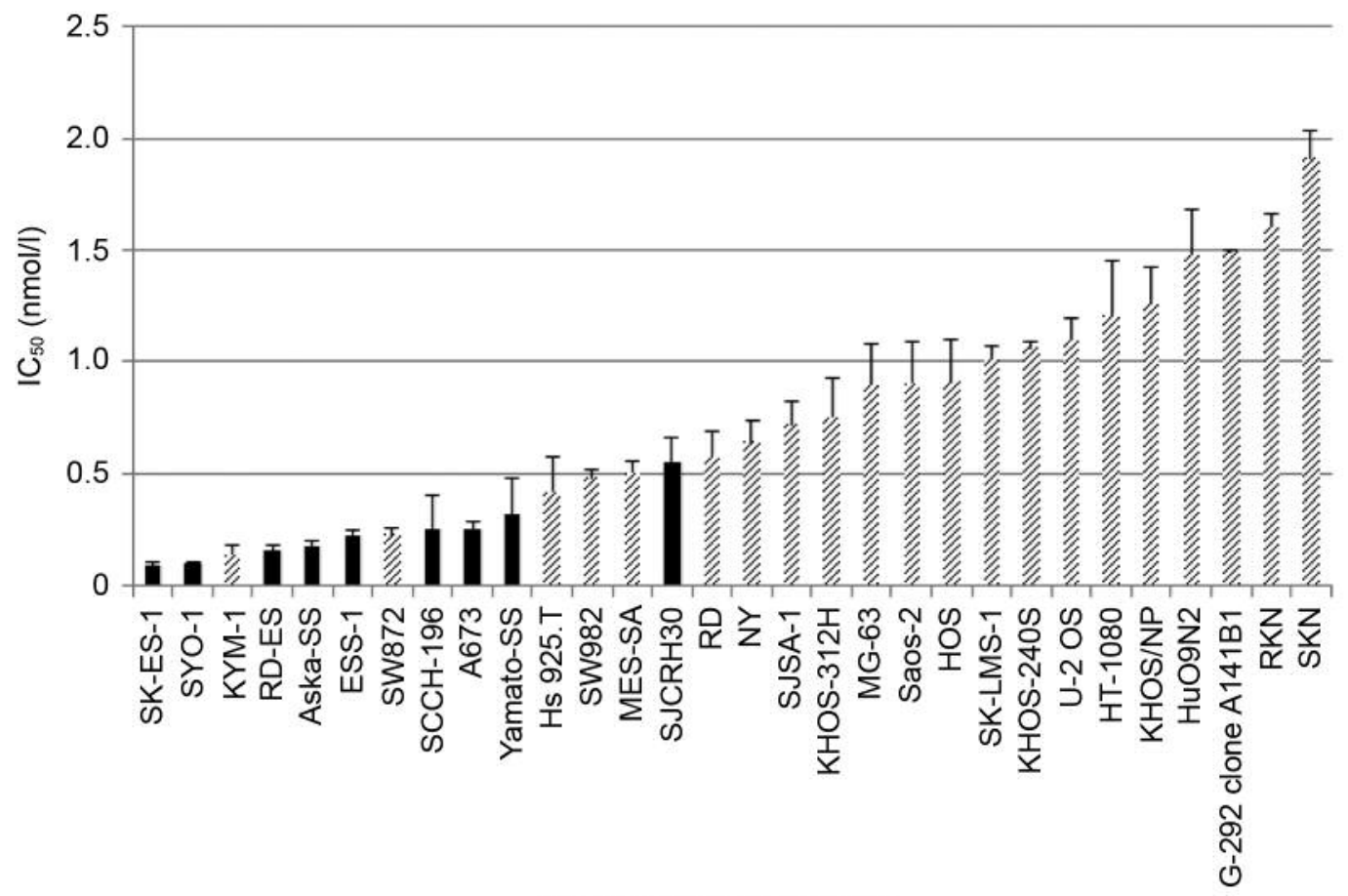

Human sarcoma cell lines

Figure 1. Trabectedin shows a significant antitumor activity in sarcoma cell lines. Thirty sarcoma cell lines were exposed to trabectedin for 72 h. Nine TRS cell lines (solid black bars) and 21 non-TRS cell lines (hashed bars) were used. The error bars represent the standard deviation (SD, $n \geq 3$ ).

mice were divided into groups using a stratified randomization method so as to equalize the mean TV in each group. Each group consisted of 6 nude mice. Trabectedin $[0.01 \mathrm{mg} / \mathrm{mL}$ in a solution of $\left.0.05 \mathrm{~mol} / 1 \mathrm{KH}_{2} \mathrm{PO}_{4}(\mathrm{pH} 4.0)\right]$ was administered by intraperitoneal bolus injection on days 1,5 , and 9 at a dose of $0.1 \mathrm{mg} / \mathrm{kg}$, which was the maximum tolerated dose in nude mice. VE- $822[30 \mathrm{mg} / \mathrm{kg}$ in a solution of $10 \%$ vitamin E tocopheryl polyethylene glycol succinate (VitE TPGS)] was administered by oral gavage on days 1,2 and 3 of each 4-day cycle. The control group was treated with the vehicle $(0.05 \mathrm{~mol} / \mathrm{l} \mathrm{KH} 2 \mathrm{PO} 4, \mathrm{pH} 4.0)$ for trabectedin on days 1,5 , and 9.

The TVs were measured twice a week until day 15. On day 15, the tumor growth inhibition rate (TGI) was calculated using the following formula: TGI $=[1-($ mean TV of treated group $) /($ mean TV of control group)] $\times 100$. The body weight change $(\mathrm{BWC}, \%)$ was calculated as [(body weight on day 15) - (body weight on day $0)] /($ body weight on day 0$) \times 100$.

The animal study was conducted according to institutional guidelines and was approved by the Institutional Animal Care and Use Committee of Taiho Pharmaceutical Co., Ltd.

Statistical analysis. The correlation analysis for the antitumor activity of trabectedin and the gene expression of SLFN11 was performed using JMP (version 9) software (SAS Institute Inc., Cary, NC, USA). The significance of the difference in the mean SLFN11 expression levels between the 2 groups (TRS $v s$. non-TRS) was analyzed using the Aspin-Welch two-tailed $t$-test. Differences were considered significant when $p<0.05$. The analysis was performed using JMP (version 9) software.
The significance of the difference in the mean SLFN11 expression levels according to decitabine treatment at each timepoint was analyzed using the Dunnett test. The significance of the difference in the mean TV between the 2 groups on day 15 was analyzed using the Aspin-Welch two-tailed $t$-test. Differences were considered significant when $p<0.05$. The analyses were performed using EXSUS version 8.0 software (CAC Croit Corp., Tokyo, Japan).

\section{Results}

Antitumor activity of trabectedin in 30 human sarcoma cell lines. To evaluate the antitumor activity of trabectedin in sarcoma cell lines, the $\mathrm{IC}_{50}$ of trabectedin was determined in both translocation related sarcoma (TRS) cell lines, which contain gene fusions, and in non-TRS cell lines using an in vitro cell viability assay. Trabectedin showed nanomolar ranges of $\mathrm{IC}_{50}$ values in both TRS and non-TRS cell lines, and the ranges of all the $\mathrm{IC}_{50}$ values were extremely narrow (Figure 1).

Expression level of SLFN11 was positively correlated with the antitumor activity of trabectedin. To determine the relationship between the SLFN11 expression levels and the sensitivity to trabectedin in 30 sarcoma cell lines, the 
a

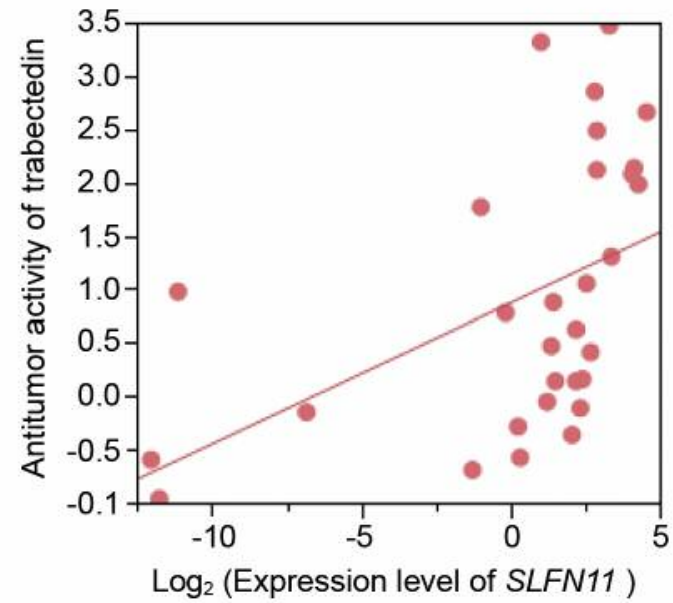

b

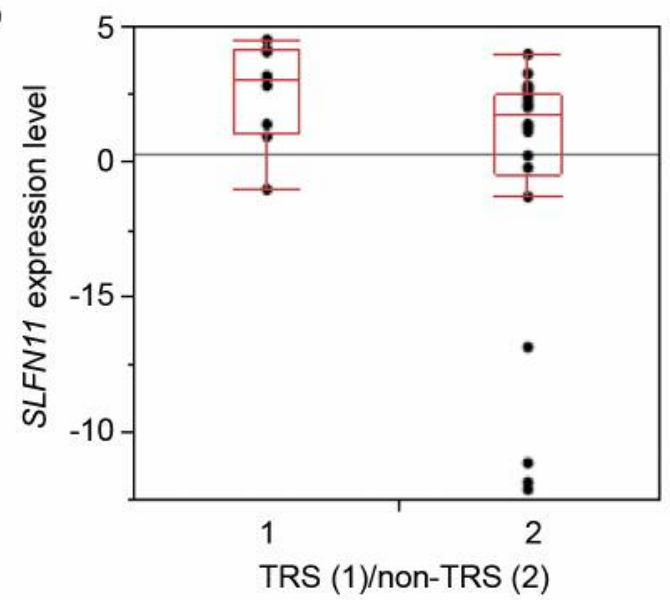

Figure 2. Antitumor activity of trabectedin was correlated with SLFN11 expression levels. (a) Correlation between SLFN11 expression (mRNA

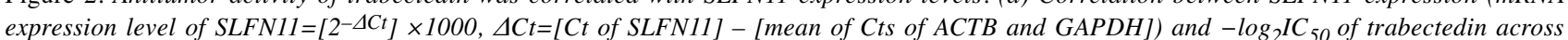
sarcoma cell lines. Correlation coefficients: $r=0.479, p<0.01$. (b) No significant difference in SLFN11 expression was seen between the TRS and non-TRS cell lines. $p=0.1203$.

a

SW 872

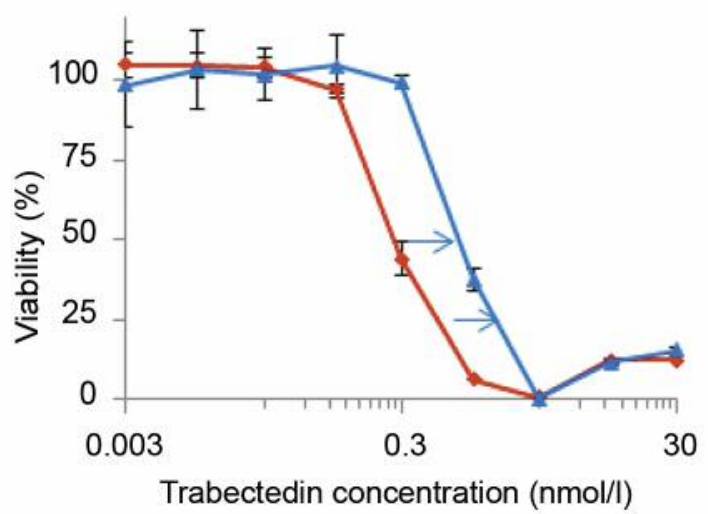

C

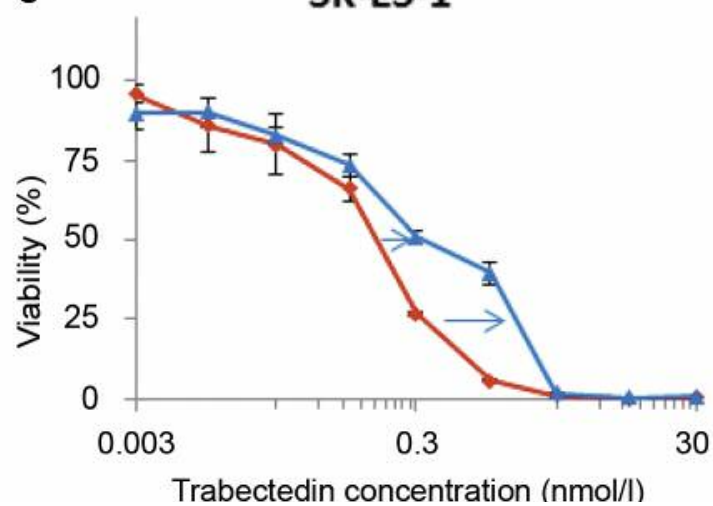

b

A673

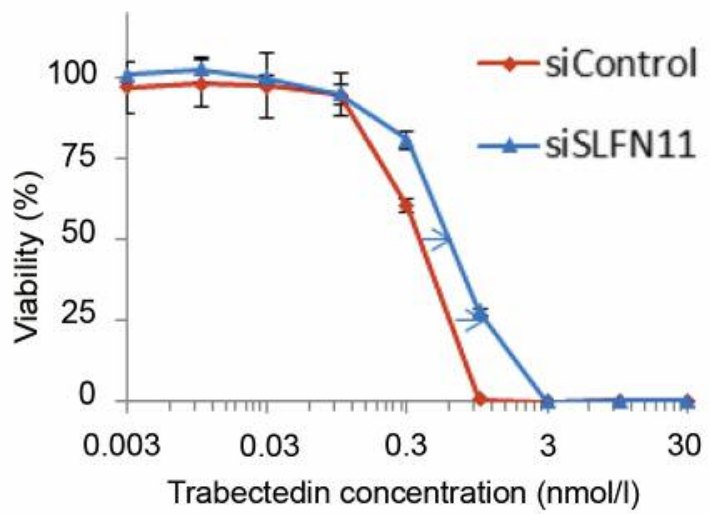

Figure 3. Antitumor activity of trabectedin was decreased in SLFN11knockdown cells. Transfection using control siRNA (siControl) and SLFN11 siRNA (siSLFN11) was performed in the indicated cell lines. The viability curves for the indicated cell lines after continuous treatment with trabectedin for $72 \mathrm{~h}$ are shown. The viability of untreated cells was set at $100 \%$. The error bars represent the standard deviation ( $S D, n \geq 3$ ). (a) $S W 872$ is a liposarcoma cell line (non-TRS). (b) A673 is a Ewing's sarcoma cell line (TRS). (c) SK-ES-1 is a Ewing's sarcoma cell line (TRS). 


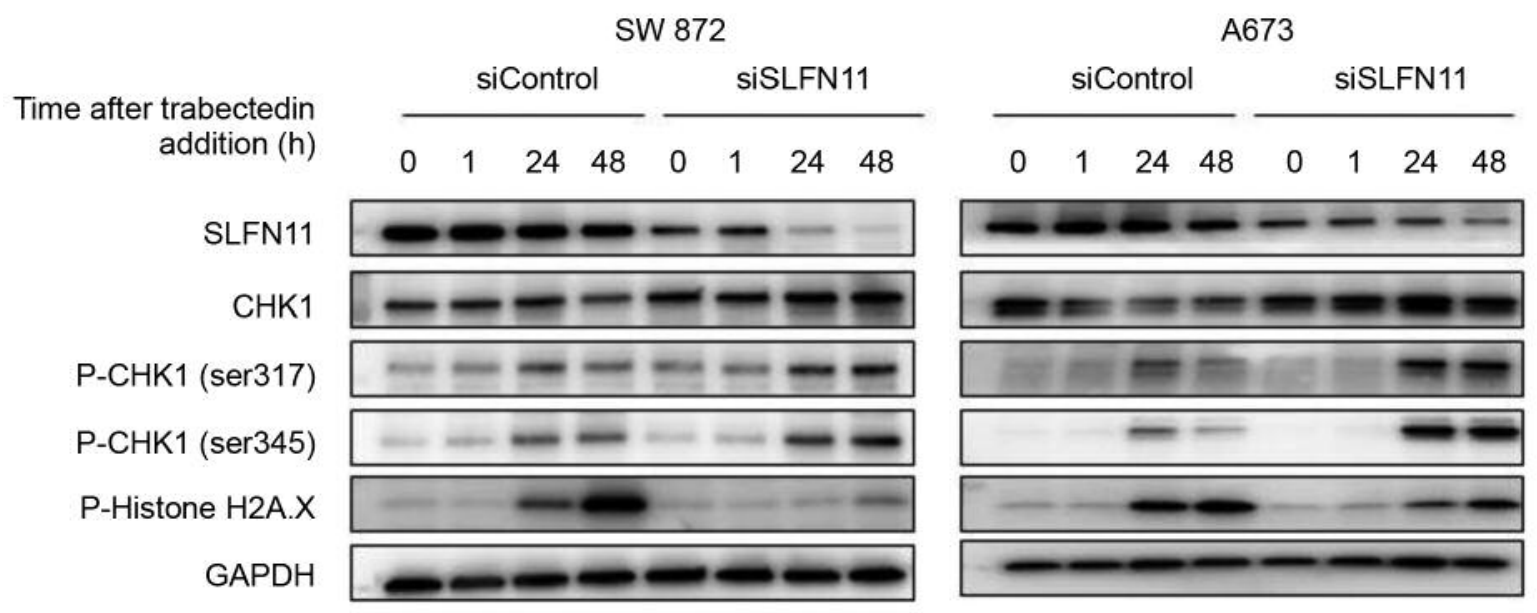

Figure 4. DNA damage checkpoint was activated by trabectedin in SLFN11-knockdown cells. SW 872 and A673 cells, which were transfected with siControl and siSLFN11, were exposed for $1 \mathrm{~h}$ with trabectedin $(20 \mathrm{nmol} / \mathrm{l})$ followed by 24 and $48 \mathrm{~h}$ post-incubation in drug-free media, as indicated. The phosphorylation of CHK1 was upregulated in all cell lines, and CHK1 was degradated in the control cells.

correlation between the antitumor activity $\left(-\log _{2} \mathrm{IC}_{50}\right)$ of trabectedin and the SLFN11 expression levels was determined using quantitative PCR in each cell line. The expression level of SLFN11 was positively correlated with the antitumor activity of trabectedin, and cell lines with high levels of SLFN11 expression were more sensitive to trabectedin (correlation coefficients: $\mathrm{r}=0.479, p<0.01$ ) (Figure 2a). Furthermore, no significant difference was seen in the gene expression levels of TRS and non-TRS cell lines (Figure 2b).

Differences in antitumor activity of trabectedin between high and low SLFN11 expression cells. To determine the causal relation of SLFN11 and trabectedin sensitivity, SLFN11 was knocked down using siRNA in three high SLFN11 expressing cell lines (liposarcoma SW 872, Ewing's sarcoma A673 and SK-ES-1) and determined the $\mathrm{IC}_{50}$ and $\mathrm{IC}_{75}$ values using cell viability assays. All three SLFN11-knockdown cell lines had a lower sensitivity to trabectedin than the control cells, which were treated with negative control siRNA (siControl). The $\mathrm{IC}_{50}$ and $\mathrm{IC}_{75}$ values of the knockdown cells were 3.4 and 2.8 fold (SW 872), 1.8 and 2.2-fold (A673), and 2.6 and 3.6-fold (SK-ES-1) higher than those of each control group, respectively (Figure 3a, b, c).

Furthermore, the SW 872 and A673 cell lines, which were transfected with siSLFN11 and siControl cells, were exposed to $20 \mathrm{nmol} / 1$ of trabectedin for $1 \mathrm{~h}$, followed by $48 \mathrm{~h}$ postincubation in drug-free media. The phosphorylation of checkpoint kinase 1 (CHK1) on Ser317 and Ser345 residues after trabectedin exposure were observed in both cells independently of SLFN11 expression levels. On the other hand, CHK1 expression was reduced in the high SLFN11 expression cells. Additionaly, the phosphorylation of Histone H2A.X after trabectedin exposure was lower in the SLFN11knockdown cells, compared with high SLFN11 expression cells (Figure 4).

Quantitative PCR analysis indicated that the U-2 OS (osteosarcoma) cell line expressed minimal levels of SLFN11. In public data from the CellMiner portal site (https://discover.nci.nih.gov/cellminercdb/), the SLFN11 gene was hypermethylated in the U-2 OS cell line, compared with other high SLFN11 expression sarcoma cell lines (Figure 5a). Furthermore, treatment of the U-2 OS cell line with $1 \mu \mathrm{mol} / 1$ decitabine restored the SLFN11 expression levels (Figure 5b). To determine whether a DNA methyltransferase inhibitor enhances the trabectedin antitumor activity in SLFN11-hypermethylated cells, the U-2 OS cell line was exposed to $1 \mu \mathrm{mol} / 1$ of decitabine for $24 \mathrm{~h}$ before adding trabectedin for $72 \mathrm{~h}$ and assaying cell viability. The $\mathrm{IC}_{50}$ value of decitabine in combination with trabectedin was approximately 2 -fold lower than that of trabectedin alone (Figure 5c).

ATR inhibitor enhanced the antitumor activity of trabectedin in SLFN11-knockdown cells. Because SLFN11 blocks replication regardless of ATR-CHK1 activity (22), in low SLFN11 expression cells, DNA damage response might be dependent on ATR. Therefore, the combined antitumor activity of an ATR inhibitor (VE-821) and trabectedin was evaluated. SW 872 and A673 cell lines, which were transfected with siSLFN11 or siControl, were exposed to $1 \mu \mathrm{mol} / 1$ of $\mathrm{VE}-821$ and trabectedin for $72 \mathrm{~h}$ (Figure $6 \mathrm{a}$ and b). As a result, VE821 enhanced the antitumor activities of trabectedin by 2 -fold in the two SLFN11-knockdown cell lines. 
a

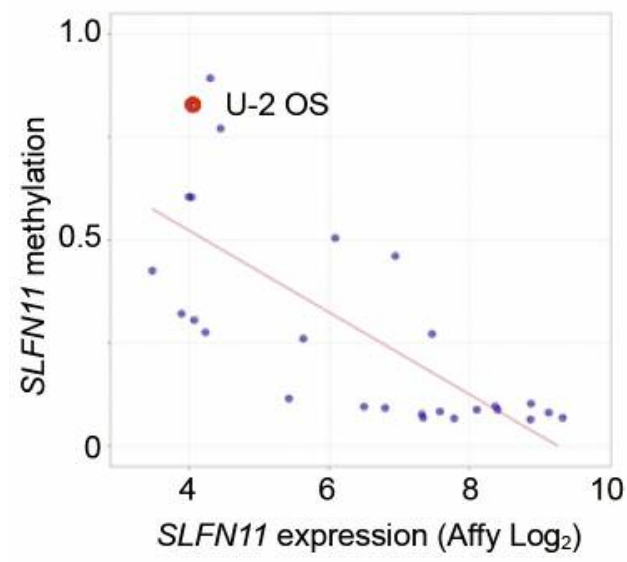

b

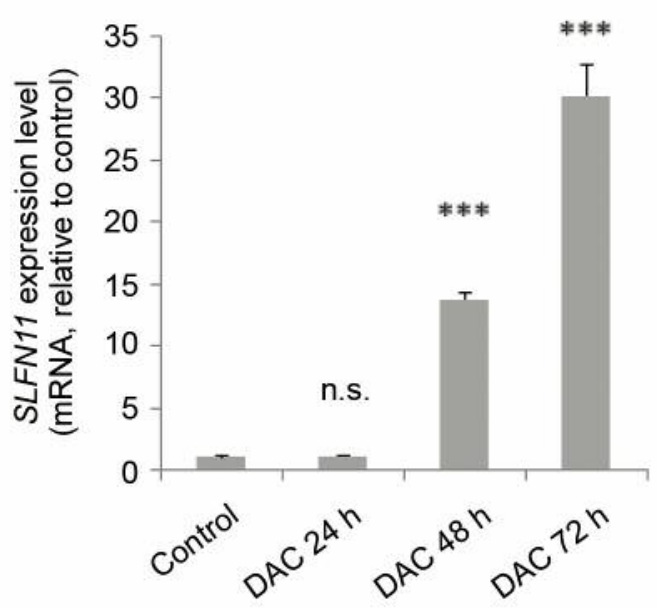

C

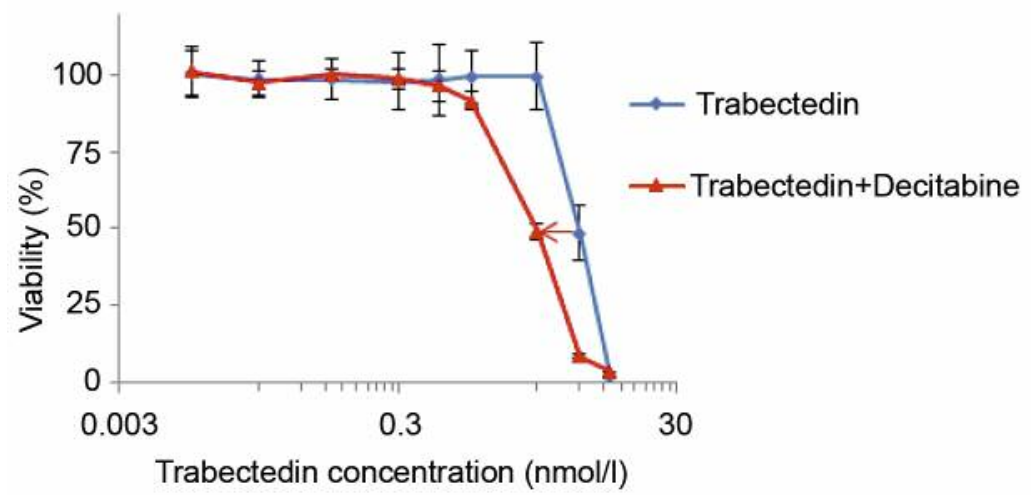

Figure 5. Demethylation of SLFN11 enhanced the antitumor activity of trabectedin. (a) SLFN11 expression in the cancer cell lines of the CCLE collection matched with promoter methylation determined from data obtained from the GDSC database for individual common cell lines across the two databases (blue dots) obtained using CellMiner (http://discover.nci.nih.gov/cellminercdb/). The U-2 OS cell line does not express SLFN11 and exhibits promoter methylation; the U-2 OS cell line is marked in red. (b) The expression of the SLFN11 RNA transcript was restored in the methylated U-2 OS cell line by treatment with the demethylating drug 5-aza-2'-deoxycytidine (DAC, decitabine). n.s: Not significant, $* * * p<0.001$. (c) Viability curves of the indicated cell lines after continuous treatment for $72 \mathrm{~h}$ with trabectedin alone (blue) and after exposure to 1 umol/l of decitabine for $24 \mathrm{~h}$ before the addition of trabectedin (red). The viability of untreated cells was set at 100\%. The error bars represent the standard deviation (SD, $n \geq 3)$.

ATR inhibitor enhanced the antitumor activity of trabectedin in a SLFN11-knockout SW 872 xenograft model. Next, the combined antitumor activity of trabectedin and ATR inhibitor (VE-822) was examined in xenograft nude mice models of SW 872 and SLFN11-knockout SW 872 (Figure 7a and b). The SLFN11 protein expression levels in each SW 872 tumor fragment used in this study were examined by western blotting (Figure $7 \mathrm{c}$ ). Mice were treated with trabectedin at 0.1 $\mathrm{mg} / \mathrm{kg} / \mathrm{day}$ (dosed by intraperitoneal bolus injection on days 1 , 5 , and 9) and VE-822 at $30 \mathrm{mg} / \mathrm{kg} /$ day (dosed by oral gavage on days 1,2, and 3 of each 4-day cycle) or a combination of both agents together. Trabectedin monotherapy induced significant antitumor activity (83\% TGI) in the high SLFN11 expression SW 872 xenograft model on Day 15. Although trabectedin monotherapy had marginal antitumor activity $(37 \%$ TGI) in the SLFN11-knockout SW 872 xenograft model, the combination with VE-822 induced a higher antitumor activity, compared with trabectedin monotherapy. The combination was well tolerated, and no increase in body weight loss was observed compared with trabectedin monotherapy.

\section{Discussion}

To clarify the role of SLFN11 in the antitumor mechanism of trabectedin, the relation between SLFN11 expression levels and the antitumor activities of trabectedin were evaluated. We demonstrated, for the first time, the role of SLFN11 in cellular responses to trabectedin. Our study 
a
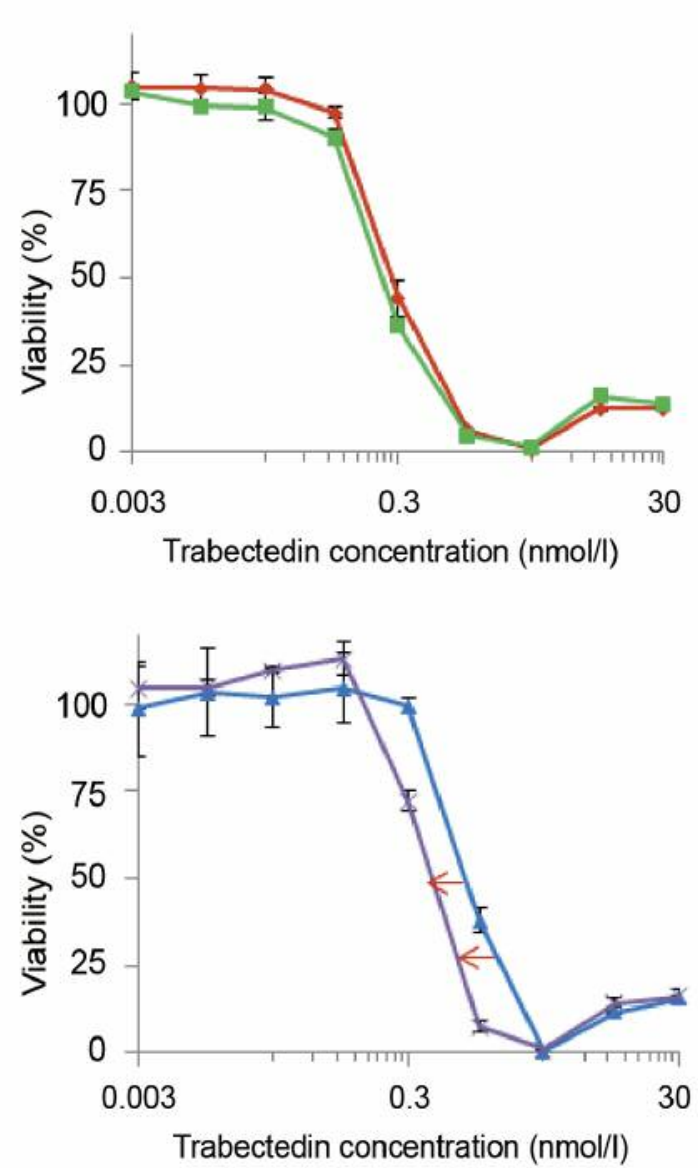

b
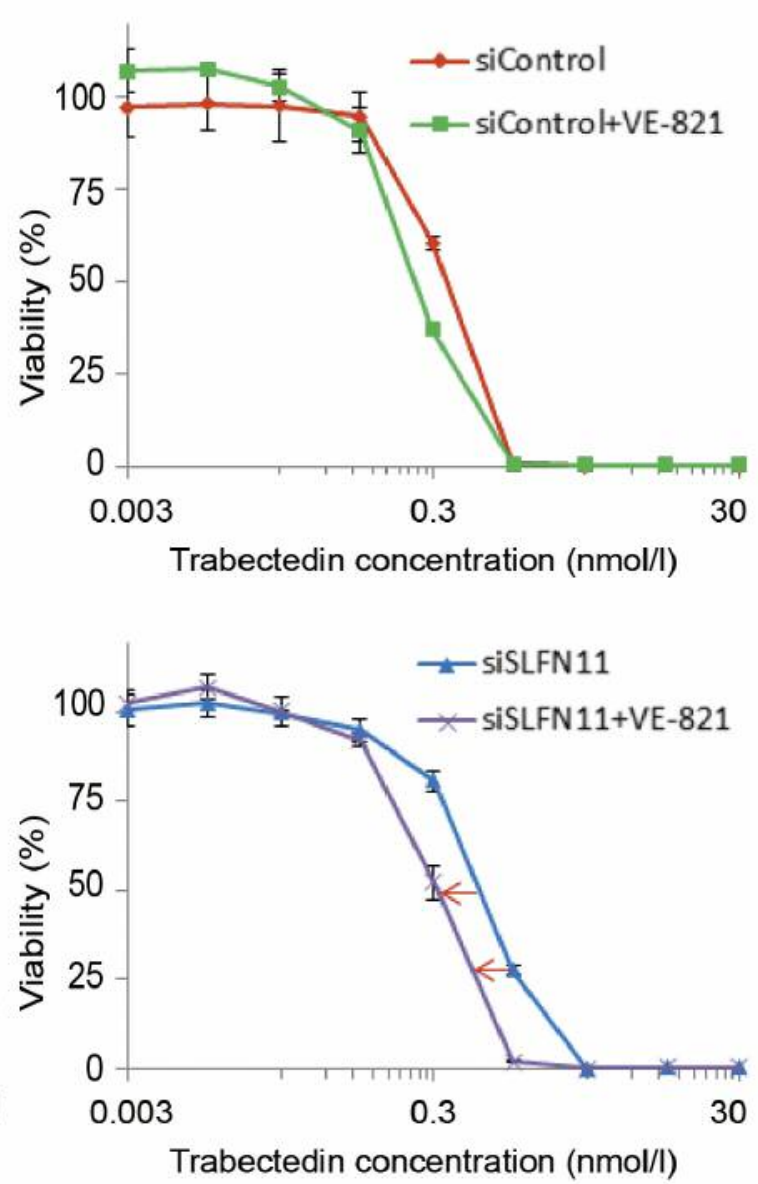

Figure 6. ATR inhibitor enhanced the antitumor activity of trabectedin in SLFN11-knockdown sarcoma cells in vitro. High SLFN11 expression (a) SW 872 and (b) A673 cell lines transfected with siControl (upper) or siSLFN11 (lower) were exposed to trabectedin alone or a combination of 1 umol/l of $V E-821$ and trabectedin for $72 \mathrm{~h}$. The viability of untreated cells was set at 100\%. Error bars represent the standard deviation (SD, $n \geq 3)$.

showed that trabectedin had an $\mathrm{IC}_{50}$ in the nanomolar range in both TRS and non-TRS cell lines, and SLFN11 expression was correlated with sensitivity to trabectedin. The antitumor mechanism of trabectedin through the function of SLFN11 seems to act independently of fusion genes. Therefore, we focused on the role of SLFN11 in the antitumor activities of trabectedin.

In this study, SLFN11-knockdown cell lines and a xenograft model of $S L F N 11$-knockout cells showed a lower sensitivity to trabectedin, compared to their parental high SLFN11 expression cells, in vitro and in vivo, respectively. In aggremment, the sensitivities to other DNA-damaging anticancer drugs such as topotecan, irinotecan, and cisplatin are also reportedly linked to $S L F N 11$ expression (19). On the other hand, SLFN11 expression did not exhibit any association with the response to drugs targeting other components, such as paclitaxel (data not shown). Since the enhancement of the antitumor activities through the modification of SLFN11 expression levels seems to be specific to DNA-damaging anticancer drugs (19), we hypothesized that SLFN11 might be involved in DNA repair. Although one group has reported a significant combined antitumor activities of trabectedin and ATR inhibitors in ovarian cancer cell lines (23), our results demonstrated that an ATR inhibitor enhanced the antitumor activity of trabectedin in SLFN11-knockdown cell lines in vitro and in SLFN11-knockout tumors in vivo. The above study showed that the ATR/CHK1 and ATM/CHK2 pathways were activated in response to trabectedin, and that the dual inhibition of ATR and ATM increased the antitumor activity of trabectedin. Furthermore, lack of SLFN11 expression is a major cause of resistance to PARP inhibitors, and addition 

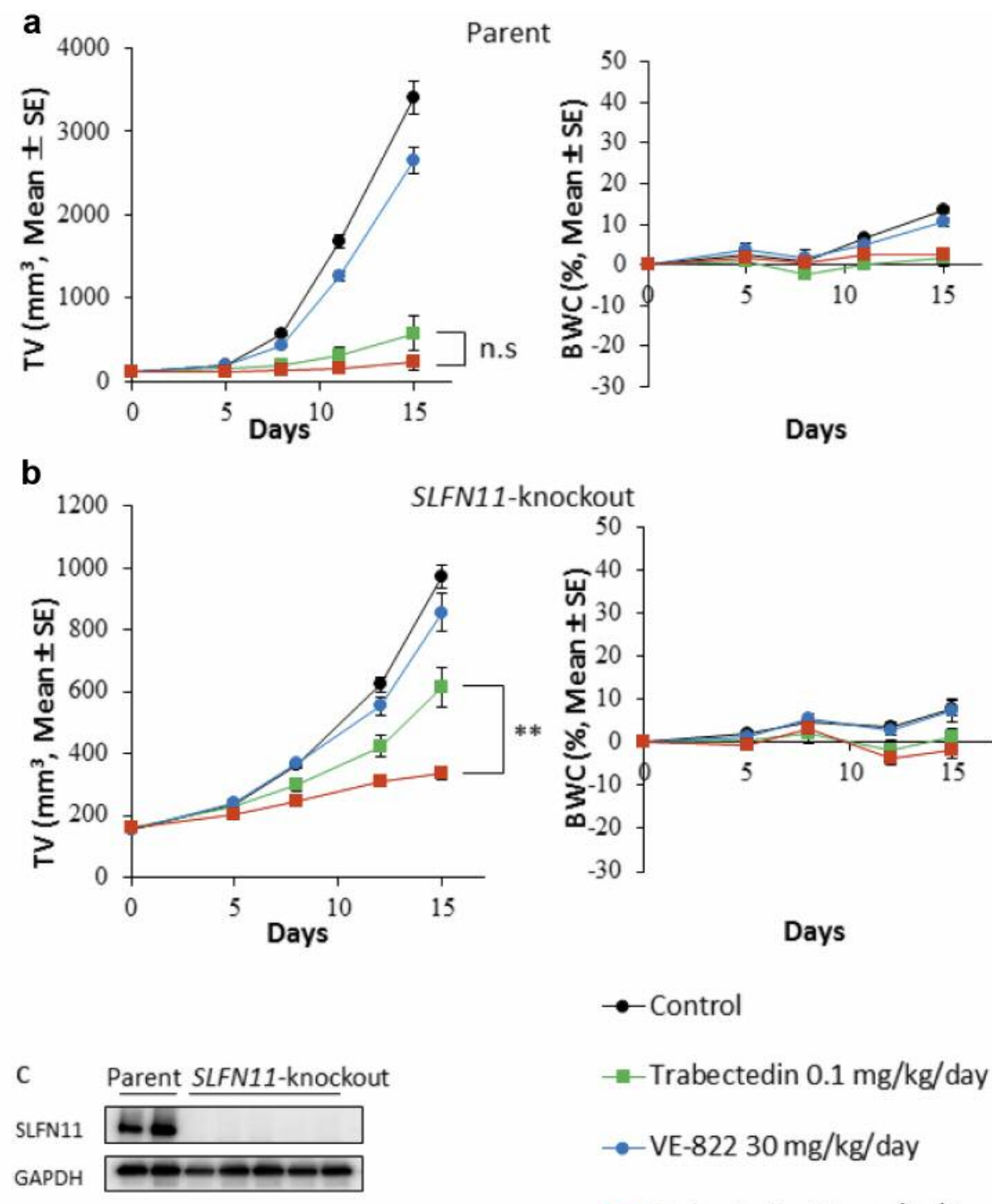

- Control

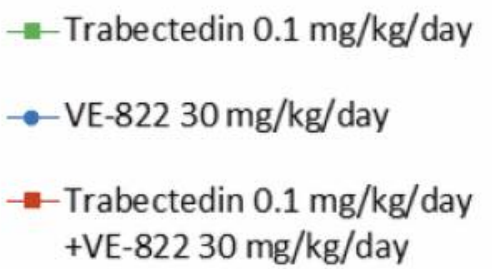

Figure 7. ATR inhibitor enhanced the antitumor activity of trabectedin in SLFN11-knockout sarcoma xenografts in vivo. A mouse xenograft experiment using (a) SW 872 (high SLFN11 expression) and (b) SLFN11-knockout SW 872 cell lines was performed. Mice bearing tumors were treated with the vehicle (dosed on days 1, 5, and 9), trabectedin (dosed on days 1, 5, and 9), VE-822 (dosed on days 1, 2, and 3 of each 4-day cycle), or a combination of both drugs. The tumor volume (TV, left) and the relative body weight change (BWC, right) were plotted. The error bars represent the SE (n=6). n.s: Not significant, ${ }^{* *} p<0.01$. (c) The lack of SLFN11 protein expression in the SLFN11-knockout SW 872 tumor fragments used in this study was examined using western blotting.

of an ATR inhibitor overcomes such resistance (24). On the other hand, the antitumor mechanism of trabectedin does not necessarily involve only DNA-damaging effects, but other effects such as the suppression of transcription factors, cellcycle regulation, or tumor microenvironment regulation might also be involved $(10,16,25)$. Therefore, SLFN11knockdown cell lines did not show resistance to trabectedin, but rather exhibited a lower sensitivity to trabectedin, unlike other DNA-damaging anticancer drugs.

In addition, a previously reported analysis of DNA methylation using the cancer cell line panel NCI60 has identified SLFN11 CpG promoter island hypermethylation as a predictive biomarker of platinum resistance $(18,26)$. Some studies have revealed that the DNA methyltransferase 
a

\section{SLFN11 high expression cells}

Trabectedin

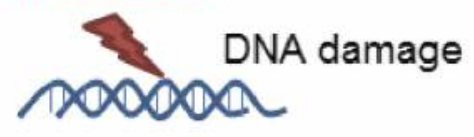

b

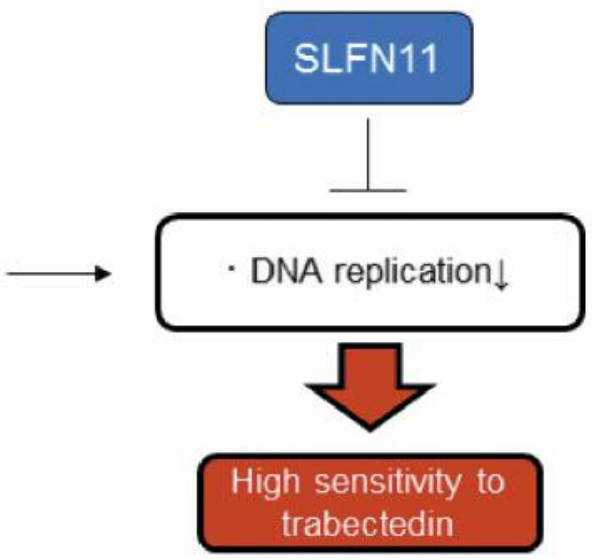

\section{SLFN11 low expression cells}

+ ATR Inhibitor

(e.g. berzosertib)

Trabectedin

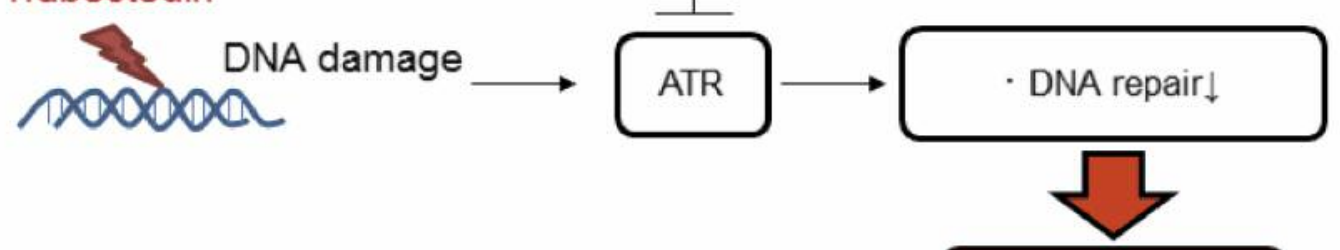

High sensitivity to

trabectedin

C

\section{SLFN11 low expression cells}

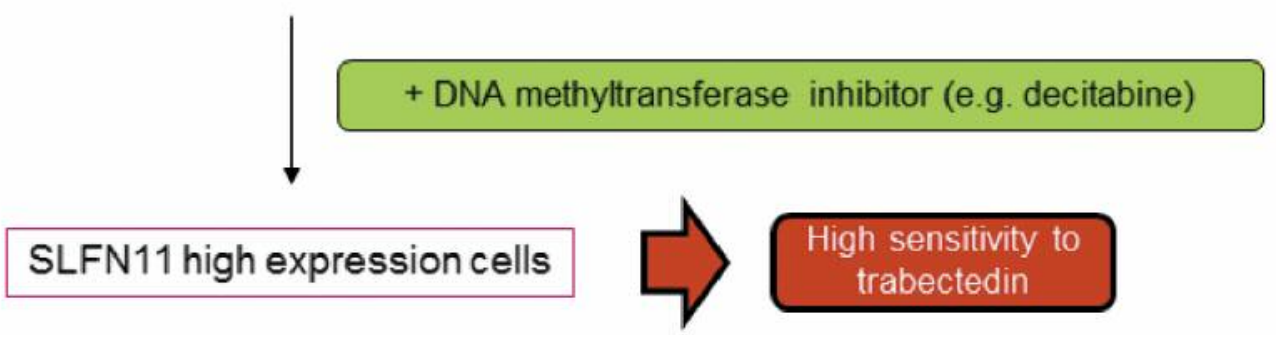

Figure 8. Speculated mechanism of the combined effect of trabectedin and an ATR inhibitor, berzosertib, or a DNA methyltransferase inhibitor, decitabine, in low-SLFN11 expression cells. (a) In high-SLFN11 expression cells, when trabectedin is added to the cells, SLFN11 might block replication (22). Therefore, trabectedin has a high antitumor activity in these cells. (b) On the other hand, DNA damage response might be dependented on ATR in low-SLFN11 expression cells, the inhibition of the DNA repair system by the ATR inhibitor berzosertib leads to enhancement of the antitumor activity of trabectedin. (c) The restoration of SLFN11 expression by the DNA methyltransferase inhibitor decitabine also leads to enhancement of the antitumor activity of trabectedin. Therefore, a combination therapy of trabectedin and berzosertib or decitabine is promising against low-SLFN11 expression tumors. 
inhibitor and the EZH2 inhibitor induce $S L F N 11$ expression in several cancer cell lines $(18,27)$. Induction of SLFN11 expression by decitabine in a sarcoma cell line and the enhancement of the antitumor activity of trabectedin by combination with decitabine were observed in the present study. These results suggest that combination treatment consisting of DNA methyltransferase inhibitor and DNAdamaging anticancer drugs, including trabectedin, could be a promising treatment against SLFN11-hypermethylated cells (Figure 8).

Recently, Murai et al. have shown that SLFN11 is recruited to stressed replication forks carrying extended RPA filaments where it blocks replication by changing the chromatin structure across replication sites independently of HRR (22). This might be the reason why the antitumor activity of trabectedin in high SLFN11 expression cells shows a higher sensitivity to trabectedin, compared with SLFN11-deficient cells. Furthermore, the report suggests that in a DNA repair system, such as HRR, DNA damage response might be dependented on ATR in low SLFN11 expression cells, and the combination of DNA-damaging anticancer drugs including trabectedin and ATR inhibitors seems promising against SLFN11-deficient cells (Figure 8).

The present study suggests the importance of SLFN11 expression as a key factor in the antitumor activity of trabectedin. Furthermore, the combination of trabectedin and an ATR inhibitor or DNA methyltransferase inhibitor was useful against SLFN11-deficient cells, in which trabectedin has a low antitumor activity.

\section{Conflicts of Interest}

Junya Iwasaki, Toshiharu Komori, Fumio Nakagawa, Hideki Nagase, Junji Uchida, and Kenichi Matsuo are employees of Taiho Pharmaceutical Co., Ltd. Yoshihiro Uto has no potential conflicts of interest to report.

\section{Authors' Contributions}

JI and TK designed the study. JI, TK and FN performed experiments. JI analyzed the data and draft the manuscript. HN, JU, $\mathrm{KM}$ and YU supervised the work and helped draft and revise the manuscript. All Authors read and approved the final manuscript.

\section{References}

1 Demetri GD, von Mehren M, Jones RL, Hensley ML, Schuetze SM, Staddon A, Milhem M, Elias A, Ganjoo K, Tawbi H, Van Tine BA, Spira A, Dean A, Khokhar NZ, Park YC, Knoblauch RE, Parekh TV, Maki RG and Patel SR: Efficacy and safety of trabectedin or dacarbazine for metastatic liposarcoma or leiomyosarcoma after failure of conventional chemotherapy: Results of a phase iii randomized multicenter clinical trial. J Clin Oncol 34(8): 786-793, 2016. PMID: 26371143. DOI: 10.1200/ JCO.2015.62.4734
2 Runnebaum IB, Reichert D, Ringsdorf U, Kuther M, Hesse T, Sehouli $J$ and Wimberger P: Trabectedin plus pegylated liposomal doxorubicin (pld) for patients with platinum-sensitive recurrent ovarian cancer: A prospective, observational, multicenter study. J Cancer Res Clin Oncol 144(6): 1185-1195, 2018. PMID: 29623421. DOI: 10.1007/s00432-018-2637-1

3 Blum JL, Goncalves A, Efrat N, Debled M, Conte P, Richards PD, Richards D, Lardelli P, Nieto A, Cullell-Young M and Delaloge S: A phase ii trial of trabectedin in triple-negative and her2-overexpressing metastatic breast cancer. Breast Cancer Res Treat 155(2): 295-302, 2016. PMID: 26749361. DOI: 10.1007/ s10549-015-3675-x

4 Colmegna B, Uboldi S, Frapolli R, Licandro SA, Panini N, Galmarini CM, Badri N, Spanswick VJ, Bingham JP, Kiakos K, Erba E, Hartley JA and D'Incalci M: Increased sensitivity to platinum drugs of cancer cells with acquired resistance to trabectedin. Br J Cancer 113(12): 1687-1693, 2015. PMID: 26633559. DOI: 10.1038/bjc.2015.407

5 Takebayashi Y, Pourquier P, Zimonjic DB, Nakayama K, Emmert S, Ueda T, Urasaki Y, Kanzaki A, Akiyama SI, Popescu $\mathrm{N}$, Kraemer KH and Pommier Y: Antiproliferative activity of ecteinascidin 743 is dependent upon transcription-coupled nucleotide-excision repair. Nat Med 7(8): 961-966, 2001. PMID: 11479630. DOI: $10.1038 / 91008$

6 Herrero AB, Martin-Castellanos C, Marco E, Gago F and Moreno $\mathrm{S}$ : Cross-talk between nucleotide excision and homologous recombination DNA repair pathways in the mechanism of action of antitumor trabectedin. Cancer Res 66(16): 8155-8162, 2006. PMID: 16912194. DOI: 10.1158/0008-5472.CAN-06-0179

7 Soares DG, Escargueil AE, Poindessous V, Sarasin A, de Gramont A, Bonatto D, Henriques JA and Larsen AK: Replication and homologous recombination repair regulate DNA double-strand break formation by the antitumor alkylator ecteinascidin 743 . Proc Natl Acad Sci USA 104(32): 13062-13067, 2007. PMID: 17656556. DOI: 10.1073/pnas.0609877104

8 Tavecchio M, Simone M, Erba E, Chiolo I, Liberi G, Foiani M, D'Incalci $\mathrm{M}$ and Damia G: Role of homologous recombination in trabectedin-induced DNA damage. Eur J Cancer 44(4): 609618, 2008. PMID: 18243687. DOI: 10.1016/j.ejca.2008.01.003

9 Erba E, Bergamaschi D, Bassano L, Damia G, Ronzoni S, Faircloth GT and D'Incalci M: Ecteinascidin-743 (et-743), a natural marine compound, with a unique mechanism of action. Eur J Cancer 37(1): 97-105, 2001. PMID: 11165136.

10 Simoens C, Korst AE, De Pooter CM, Lambrechts HA, Pattyn GG, Faircloth GT, Lardon F and Vermorken JB: In vitro interaction between ecteinascidin 743 (et-743) and radiation, in relation to its cell cycle effects. Br J Cancer 89(12): 2305-2311, 2003. PMID: 14676811. DOI: 10.1038/sj.bjc.6601431

11 Nam EA and Cortez D: Atr signalling: More than meeting at the fork. Biochem J 436(3): 527-536, 2011. PMID: 21615334. DOI: 10.1042/BJ20102162

12 Zeman MK and Cimprich KA: Causes and consequences of replication stress. Nat Cell Biol 16(1): 2-9, 2014. PMID: 24366029. DOI: $10.1038 / \mathrm{ncb} 2897$

13 Josse R, Martin SE, Guha R, Ormanoglu P, Pfister TD, Reaper PM, Barnes CS, Jones J, Charlton P, Pollard JR, Morris J, Doroshow JH and Pommier Y: Atr inhibitors ve-821 and vx-970 sensitize cancer cells to topoisomerase i inhibitors by disabling DNA replication initiation and fork elongation responses. Cancer Res 74(23): 6968-6979, 2014. PMID: 25269479. DOI: 10.1158/ 0008-5472.CAN-13-3369 
14 Karnitz LM and Zou L: Molecular pathways: Targeting atr in cancer therapy. Clin Cancer Res 21(21): 4780-4785, 2015. PMID: 26362996. DOI: 10.1158/1078-0432.CCR-15-0479.

15 Mertens F, Antonescu CR, Hohenberger P, Ladanyi M, Modena P, D'Incalci $M$, Casali PG, Aglietta $M$ and Alvegard T: Translocation-related sarcomas. Semin Oncol 36(4): 312-323, 2009. PMID: 19664492. DOI: 10.1053/j.seminoncol. 2009.06.004

16 Friedman D, Hu Z, Kolb EA, Gorfajn B and Scotto KW: Ecteinascidin-743 inhibits activated but not constitutive transcription. Cancer Res 62(12): 3377-3381, 2002. PMID: 12067978

17 Grohar PJ, Griffin LB, Yeung C, Chen QR, Pommier Y, Khanna $\mathrm{C}$, Khan $\mathrm{J}$ and Helman LJ: Ecteinascidin 743 interferes with the activity of ews-fli1 in ewing sarcoma cells. Neoplasia 13(2): 145-153, 2011. PMID: 21403840.

18 Nogales V, Reinhold WC, Varma S, Martinez-Cardus A, Moutinho C, Moran S, Heyn H, Sebio A, Barnadas A, Pommier $\mathrm{Y}$ and Esteller $\mathrm{M}$ : Epigenetic inactivation of the putative DNA/rna helicase slfn11 in human cancer confers resistance to platinum drugs. Oncotarget 7(3): 3084-3097, 2016. PMID: 26625211. DOI: 10.18632 oncotarget.6413

19 Zoppoli G, Regairaz M, Leo E, Reinhold WC, Varma S, Ballestrero A, Doroshow JH and Pommier Y: Putative DNA/rna helicase schlafen-11 (slfn11) sensitizes cancer cells to DNAdamaging agents. Proc Natl Acad Sci USA 109(37): 1503015035, 2012. PMID: 22927417. DOI: 10.1073/pnas.1205943109

20 Barretina J, Caponigro G, Stransky N, Venkatesan K, Margolin AA, Kim S, Wilson CJ, Lehar J, Kryukov GV, Sonkin D, Reddy A, Liu M, Murray L, Berger MF, Monahan JE, Morais P, Meltzer J, Korejwa A, Jane-Valbuena J, Mapa FA, Thibault J, Bric-Furlong E, Raman P, Shipway A, Engels IH, Cheng J, Yu GK, Yu J, Aspesi P, Jr., de Silva M, Jagtap K, Jones MD, Wang L, Hatton C, Palescandolo E, Gupta S, Mahan S, Sougnez C, Onofrio RC, Liefeld T, MacConaill L, Winckler W, Reich M, Li N, Mesirov JP, Gabriel SB, Getz G, Ardlie K, Chan V, Myer VE, Weber BL, Porter J, Warmuth M, Finan P, Harris JL, Meyerson M, Golub TR, Morrissey MP, Sellers WR, Schlegel R and Garraway LA: The cancer cell line encyclopedia enables predictive modelling of anticancer drug sensitivity. Nature 483(7391): 603-607, 2012. PMID: 22460905. DOI: 10.1038/ nature 11003

$21 \mathrm{Mu}$ Y, Lou J, Srivastava M, Zhao B, Feng XH, Liu T, Chen J and Huang J: Slfn11 inhibits checkpoint maintenance and homologous recombination repair. EMBO Rep 17(1): 94-109, 2016. PMID: 26658330. DOI: 10.15252/embr.201540964
22 Murai J, Tang SW, Leo E, Baechler SA, Redon CE, Zhang H, Al Abo M, Rajapakse VN, Nakamura E, Jenkins LMM, Aladjem MI and Pommier Y: Slfn11 blocks stressed replication forks independently of atr. Mol Cell 69(3): 371-384 e376, 2018. PMID: 29395061. DOI: 10.1016/j.molcel.2018.01.012

23 Lima M, Bouzid H, Soares DG, Selle F, Morel C, Galmarini CM, Henriques JA, Larsen AK and Escargueil AE: Dual inhibition of atr and atm potentiates the activity of trabectedin and lurbinectedin by perturbing the DNA damage response and homologous recombination repair. Oncotarget 7(18): 2588525901, 2016. PMID: 27029031. DOI: 10.18632/oncotarget.8292.

24 Murai J, Feng Y, Yu GK, Ru Y, Tang SW, Shen Y and Pommier Y: Resistance to parp inhibitors by slfn11 inactivation can be overcome by atr inhibition. Oncotarget 7(47): 76534-76550, 2016. PMID: 27708213. DOI: 10.18632/oncotarget.12266

25 Germano G, Frapolli R, Belgiovine C, Anselmo A, Pesce S, Liguori M, Erba E, Uboldi S, Zucchetti M, Pasqualini F, Nebuloni M, van Rooijen N, Mortarini R, Beltrame L, Marchini S, Fuso Nerini I, Sanfilippo R, Casali PG, Pilotti S, Galmarini CM, Anichini A, Mantovani A, D'Incalci $\mathrm{M}$ and Allavena P: Role of macrophage targeting in the antitumor activity of trabectedin. Cancer Cell 23(2): 249-262, 2013. PMID: 23410977. DOI: 10.1016/j.ccr.2013.01.008

26 Reinhold WC, Varma S, Sunshine M, Rajapakse V, Luna A, Kohn KW, Stevenson H, Wang Y, Heyn H, Nogales V, Moran S, Goldstein DJ, Doroshow JH, Meltzer PS, Esteller M and Pommier Y: The nci-60 methylome and its integration into cellminer. Cancer Res 77(3): 601-612, 2017. PMID: 27923837. DOI: 10.1158/0008-5472.CAN-16-0655

27 Gardner EE, Lok BH, Schneeberger VE, Desmeules P, Miles LA, Arnold PK, Ni A, Khodos I, de Stanchina E, Nguyen T, Sage J, Campbell JE, Ribich S, Rekhtman N, Dowlati A, Massion PP, Rudin CM and Poirier JT: Chemosensitive relapse in small cell lung cancer proceeds through an ezh2-slfn11 axis. Cancer Cell 31(2): 286-299, 2017. PMID: 28196596. DOI: 10.1016/j.ccell.2017.01.006
Received February 13, 2019

Revised March 18, 2019

Accepted March 19, 2019 